\title{
MODELO RED DE BASE RADIAL PARA CLASIFICAR A LOS POBLADORES DE LOS DISTRITOS DE SAN JUAN DE LURIGANCHO, PUNO Y TARAPOTO, SEGÚN SU PERCEPCIÓN SOBRE LOS ROLES DE GÉNERO
}

\author{
Maria Estela Ponce Aruneri y Ofelia Roque Paredes
}

\section{Resumen}

El propósito de la presente investigación es modelar estadísticamente mediante el modelo red de base radial, la relación entre estilo de crianza, relaciones familiares, situación laboral y nivel educativo; para clasificar a las mujeres y hombres mayores de 14 años, de los distritos de San Juan de Lurigancho, Puno y Tarapoto, en función a su percepción sobre los roles de género, e identificar los factores que más influyen en dicha percepción. El modelo propuesto clasificó correctamente al 82,8\% de hombres y mujeres; identificó al nivel educativo, maltrato (físico y/o psicológico) cuando era niña (o) y situación laboral, como los factores que más influyen en la percepción sobre su rol de género.

Palabras clave: red de base radial; función de base radial normalizada; rol de género.

\section{Abstract}

The purpose of this research is statistically modeled by network model of radial basis, the relationship between parenting style, family relationships, employment status and educational level, to classify women and men over 14 years, from the districts of San Juan de Lurigancho , Puno and Tarapoto; based on their perception of gender roles, and identify factors that influence this perception. The model correctly classified $82,8 \%$ of men and women, identify educational level, abused (physical and / or psychological) when they were children and 
employment status, as the factors that influence the perception of their gender role.

Keywords: radial basis network; normalized radial basis function; gender role.

\section{Introducción}

La percepción es la función psíquica que permite al organismo, a través de los sentidos, recibir, elaborar e interpretar la información proveniente de su entorno.

Una diferencia importante a mencionar es la que se refiere al sexo y al género. Mientras que sexo se refiere a la dimensión biológica de ser hombre o mujer, género se refiere a las dimensiones psicológicas, sociales, conductuales y culturales.

Los roles de género son el conjunto de actividades y expectativas diferentes para mujeres y hombres que marcan la diferencia respecto a cómo ser, cómo sentir y cómo actuar. Estos roles son asignados por la sociedad en que vivimos y, como consecuencia de ello, las personas se desarrollan como mujeres o como hombres, identificándose con los roles que por su sexo le han sido asignados.

El concepto de "Roles de Género" es importante para entender algunos procesos que se interrelacionan en la vida cotidiana. Su transformación podría ser un paso importante para conseguir vivir en una sociedad más equitativa.

Las investigaciones y publicaciones sobre este tema solo vienen aplicando técnicas estadísticas univariadas y bivariadas, pero el género abarca varias dimensiones, por lo que debe ser estudiado desde un enfoque multivariado. En la actualidad, las técnicas basadas en el aprendizaje adaptativo causan gran impacto, debido a su extraordinaria aplicabilidad práctica, captando la atención de los profesionales estadísticos, los cuales comienzan a incorporar modelos y técnicas de la inteligencia artificial, como las redes neuronales artificiales al conjunto de herramientas estadísticas orientadas a la clasificación de patrones, predicción, entre otros.

Las redes neuronales artificiales constituyen una herramienta de modelización (clasificación, predicción, reducción, etc.) y análisis que se puede integrar a diversos campos, como la robótica, la ingeniería, la psicología, entre otras. Las redes neuronales tienen como objetivo obtener modelos coherentes con la realidad observada, de tal forma que son los datos los que determinan el comportamiento de la red, a través de la especificación de sus estructuras, parámetros internos, entre otros.

En problemas de clasificación, podemos encontrar numerosos ejemplos en los que las redes neuronales han mostrado con frecuencia, una mejor precisión que las técnicas estadísticas clásicas, como el análisis discriminante o la regresión logística.

En problemas reales, con frecuencia se comprueba que el análisis dis- 
criminante lineal o cuadrático, o la regresión logística, no son capaces de determinar las fronteras de decisión de un problema de clasificación cuando dichas fronteras de decisión alcanzan un alto nivel de no linealidad. Una técnica alternativa es no suponer nada acerca de las distribuciones de probabilidad de los datos y estimar directamente los coeficientes del modelo a partir de los datos de entrenamiento disponibles.

Como herramientas de predicción/ clasificación, muchos autores vienen estudiando las relaciones entre las técnicas estadísticas convencionales y los modelos de redes neuronales artificiales, entre los que tenemos a W.S. Sarle (1994), C.M. Bishop (1995), Vicino (1998). Las RNA han sido conceptualizadas como técnicas estadísticas no paramétricas, al estar libres del cumplimiento de los supuestos teóricos de la estadística paramétrica.

Para nuestra investigación aplicaremos el modelo de red de base radial, utilizando como función de activación para la capa oculta la denominada función de base radial normalizada.

Los objetivos de la presente investigación, son:

a. Construir un modelo de clasificación, utilizando la red de base radial que asigne a las mujeres y hombres mayores de 14 años de los distritos de San Juan de Lurigancho, Puno y Tarapoto, en uno de los siguientes grupos: más a los hombres, más a las mujeres, ambos por igual; en concordancia con sus percepciones acerca de los roles de género.

b. Determinar los factores influyentes en las percepciones sobre los roles de género de la población mayor de 14 años, de los distritos bajo estudio.

\section{Metodología}

\subsection{Modelo red de base radial}

Las redes neuronales de base radial (RBR) surgen con el objetivo de conseguir redes neuronales cuyo proceso de aprendizaje sea mucho más rápido que la red perceptrón multicapa.

Para ello, se diseñó una red en la que las neuronas de su única capa oculta tuvieran un carácter local, de tal forma que a la entrada de nuevos patrones hubiera menos neuronas que procesar. Esta es de hecho su principal diferencia con la red perceptrón multicapa. El comportamiento local de sus neuronas significa que cada neurona de la capa oculta se activa en una región diferente del espacio de patrones o capa de entrada.

La mayor contribución a la teoría, diseño y aplicaciones de esta red se deben a Moody y Darken en 1989, Renalds en 1989, Poggio y Girosi en 1990.

Las RBR definen hiperesferas o hiperelipses que dividen el espacio de entrada, por lo que cada neurona oculta construye una aproximación local y no lineal en una determinada región de dicho espacio. 
Figura 1: Espacio de entrada dividido por hiperferas o hiperelipses

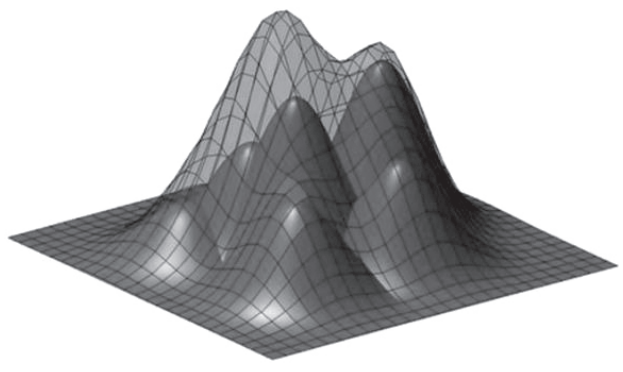

\subsection{Topologia de la red de base ra-} dial

Las redes neuronales de base radial están conformadas por una capa de neuronas de entrada, una capa de neuronas de salida y solo una capa de neuronas ocultas.

Las conexiones en este tipo de red son todas hacia adelante, es decir, la entrada de todas las neuronas de la capa oculta preceden a la salida de todas las neuronas de la capa de entrada, y a su vez las neuronas de la capa oculta transmiten su salida a todas las neuronas de la capa de salida.

La construcción de una red RBR requiere de una mayor cantidad de neuronas en los nodos ocultos que en las redes que usan backpropagation.

Las principales caracteristicas de la $R B R$ :

$>$ Capa de entrada: reciben las señales del exterior, no realizan ningún preprocesado; por ello, el peso de sus conexiones es siempre igual a uno.

> Capa Oculta: reciben las señales de la capa de entrada y realizan una transformación local y no lineal sobre dichas señales (diferencia con el modelo de red perceptrón multicapa).

> Capa de Salida: realiza una combinación lineal de las activaciones de las neuronas de la capa oculta y actúa como salida de la red; su función de activación es la función identidad.

Figura 2: Topología de la red de base radial

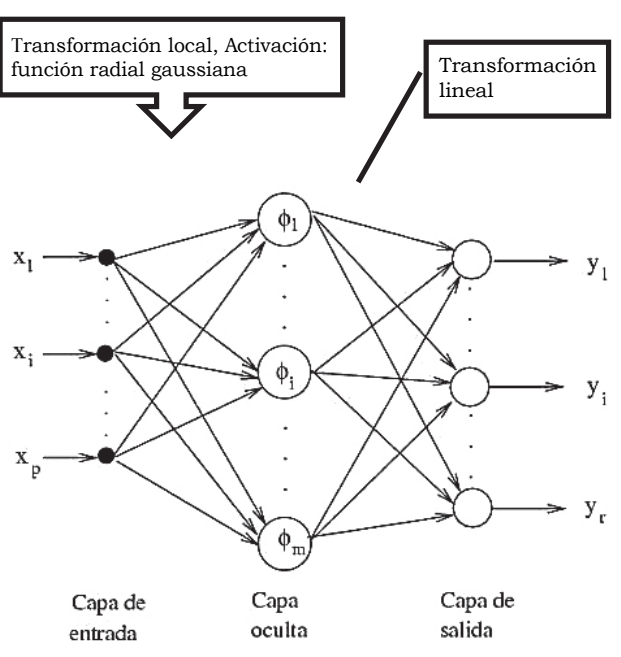

De la capa de entrada a la capa oculta no lleva ningún peso asociado; solo la neurona de salida tiene umbral.

Aunque las RBR no son comúnmente utilizadas en aplicaciones que impliquen un alto volumen de patrones de entrenamiento, se le reconoce como una red con una alta eficiencia en la fase de entrenamiento.

\subsubsection{Activación de las neuronas de la RBR}

El modelo de redes neuronales artificiales de base radial: 


$$
y_{k}^{*}(n)=\sum_{i=1}^{m} w_{i k} \phi_{i}(n)+u_{k} \quad k=1,2, \ldots, r
$$

Compuesto por $p$ neuronas en la capa de entrada, $m$ neuronas en la capa oculta, $r$ neuronas en la capa de salida, y patrón de entrada $n$.

$w_{i k}$ es el peso sináptico de la conexión entre la neurona oculta $i$ a la neurona de salida $k$;

$u_{k}$ es el umbral de la neurona de salida $k$;

$\varphi_{\mathrm{i}}(n)$ son las activaciones de las neuronas ocultas para los patrones de entrada.

La función de base radial, se define como:

$\phi_{i}(n)=\phi\left[\frac{\left\|X(n)-C_{i}\right\|}{d_{i}}\right]=\phi\left(\frac{r}{d_{i}}\right) \quad i=1,2, \ldots, m$

\section{Donde:}

$\varphi$ es la función de base radial.

$X(n)$ es el vector de entrada de la red, que constituye la capa de entrada.

$C_{i}$ son vectores que representan los centros del cluster, peso asociado a la neurona " $\imath$ ".

$d_{i}$ son números reales que representan la desviación o dilatación de la función de base radial.

$r=\left\|X(n)-C_{i}\right\|=\left[\sum_{j=1}^{p}\left(x_{j}(n)-c_{i j}\right)^{2}\right]^{0.5}$

es la distancia euclidiana del vector de entrada al centro de la función de base radial.
Algunas funciones de base radial:

$$
\begin{aligned}
& \phi(r)=e^{-\frac{r^{2}}{2}} \quad r \in \Re \\
& \phi(r)=\frac{1}{1+r^{2}} \quad r \text { } \\
& \phi(r)=\frac{1}{\sqrt{1+r^{2}}}
\end{aligned}
$$

Denominadas: funciones gaussiana, inversa cuadrática e inversa multicuadrática, respectivamente.

La función gaussiana es la más utilizada dentro de las redes neuronales de base radial y su función de activación para las neuronas de la capa oculta, es:

$$
\phi_{i}(n)=e^{-\frac{r^{2}}{2 d_{i}^{2}}} \quad i=1,2, \ldots, m
$$

Algunos paquetes estadísticos como IBM SPSS Statistics, incluyen como función de activación para las neuronas de la capa oculta, la función de activación gaussiana normalizada:

$$
\phi_{i}(n)=\frac{e^{-\frac{r^{2}}{2 d_{i}^{2}}}}{\sum_{i=1}^{m} e^{-\frac{r^{2}}{2 d_{i}^{2}}}} \quad i=1,2, \ldots, m
$$

\subsubsection{Carácter local de la RBR}

Este carácter local se debe a que dado un patrón o capa de entrada (X) a la red, sólo aquellas neuronas ocultas cuyo centro esté en la vecindad de 
dicho patrón se van activar: el resto de neuronas ocultas permanecerán inactivas o con un menor nivel de activación; es decir, a medida que el patrón se aleja del centro, el valor de la función tiende al valor mínimo de su recorrido.

Las salidas de las redes de neuronas de base radial son, por tanto, una combinación lineal de gaussianas, cada una de las cuales se activa para una determinada porción del espacio definido por los patrones de entrada.

Figura 3: Activación local de las neuronas ocultas en una red de base radial

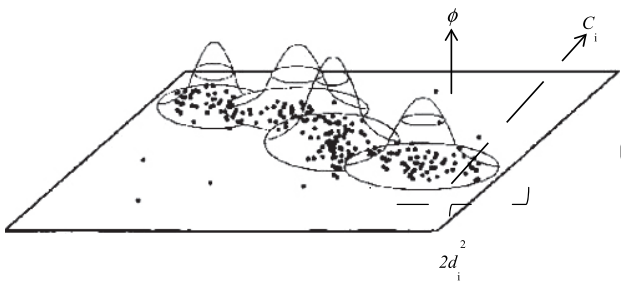

\subsubsection{Diseño de la arquitectura de la RBR}

El número de neuronas de la capa de entrada y salida de una red de base radial, está dado por el número de variables explicativas y las variables de respuesta, respectivamente. Para nuestro caso, las variables explicativas son la relación entre estilo de crianza, relaciones familiares, situación laboral y nivel educativo, y los cuatro tipos de percepciones sobre rol de género conforman las variables de respuesta.

Se realizó un análisis previo para determinar las variables más relevantes y significativas para el problema a tratar.

El número de neuronas oculta de la red se determina por pruebas y error, variando el número de neuronas hasta conseguir que la red resuelva el problema.
En una RBR, añadir o eliminar unas pocas neuronas ocultas podría influir significativamente en los resultados de la red. Es por esta situación que en los últimos años se han realizado una serie de estudios que permiten determinar en forma automática el número adecuado de neuronas ocultas y los parámetros asociados a dichas neuronas (centros y desviaciones).

\subsection{Entrenamiento o aprendizaje de la RBR}

Este proceso permite estimar los parámetros del modelo: centros y desviaciones de las neuronas ocultas, los pesos de las capas ocultas a las capas de salida, y los umbrales de las neuronas de salida.

Como las capas de neuronas en una RBR realizan tareas diferentes, se separa el proceso de optimización en dos procesos:

$>$ La determinación de los parámetros de la capa oculta, se realiza mediante la optimización en el espacio de entradas.

$>$ La determinación de los parámetros de la capa de salida, se realiza mediante la optimización en base a las salidas que se desea obtener.

Los métodos utilizados para el proceso de aprendizaje de la RBR, son: híbrido, totalmente supervisado, y un tercer método, que es la combinación de los dos anteriores.

El método híbrido es el más utilizado por los resultados satisfactorios que proporciona. 
Método de aprendizaje hibrido, tiene dos fases:

Figura 4: Fases de aprendizaje del método híbrido

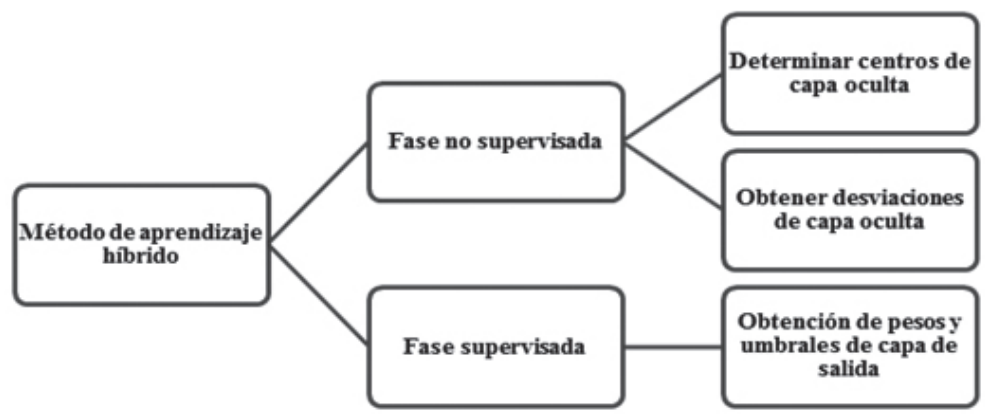

Dados los patrones de entrada y salida deseadas $\{\mathbf{X}(n), \mathbf{Y}(n)\}$, podemos resumir este método de aprendizaje en los siguientes pasos:

\section{$1^{\circ}$ Fase no supervisada:}

- Aplicar el método de k-medias al conjunto de patrones de entrada, para calcular los centros de las funciones de base radial, siendo $\mathrm{k}$ el número de neuronas ocultas en la red.

- Calcular las desviaciones de las funciones de base radial, utilizando:

- Media uniforme de las distancias euclidianas del centro a los $p$ centros más cercanos.

- Media geométrica de la distancia del centro a sus vecinos más cercanos.

Figura 5: Fase no supervisada, de aprendizaje del método híbrido

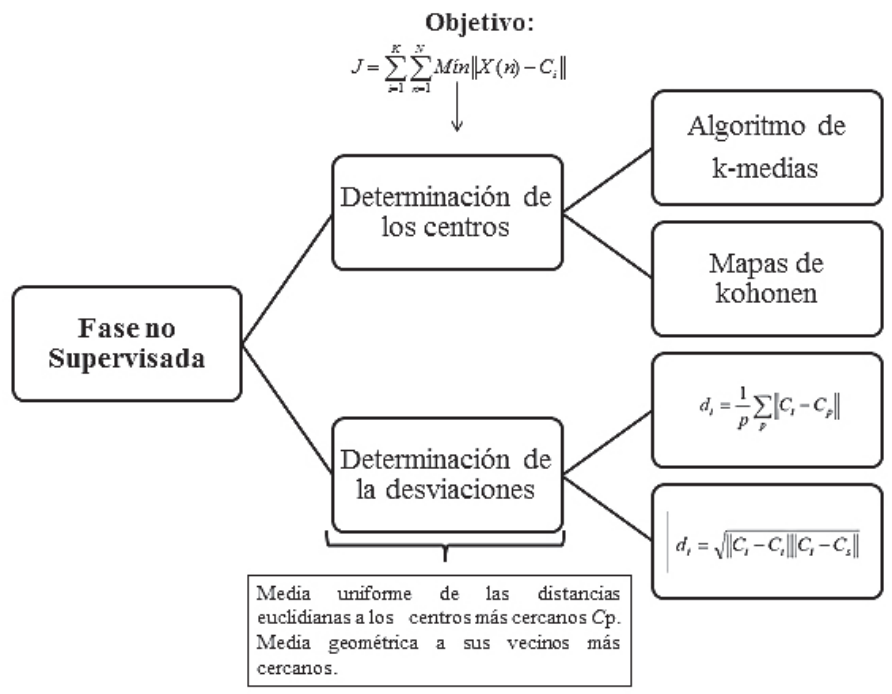




\section{$2^{\circ}$ Fase supervisada}

- Se determinan los pesos y umbrales de la capa de salida, mediante el siguiente proceso iterativo:

$\checkmark$ Los valores iniciales de los pesos y umbrales de la capa de salida, se asignan aleatoriamente.

$\checkmark$ Tomar un patrón del conjunto $\{\mathrm{X}(\mathrm{n}), \mathrm{Y}(\mathrm{n})\}$, y se calcula la salida de la red $Y^{*}(\mathrm{n})$ para el patrón de entrada $X(n)$.

$\checkmark$ Evaluar el error cometido por la red para dicho patrón "e(n)".

$\checkmark$ Se modifican los parámetros de la red, utilizando las si- guientes reglas de aprendizaje:

$$
w_{i k}(n)=w_{i k}(n-1)+\alpha_{1}\left(y_{k}^{*}(n)-y_{k}(n)\right) \phi_{i}(n)
$$$$
u_{k}(n)=u_{k}(n-1)+\alpha_{1}\left(y_{k}^{*}(n)-y_{k}(n)\right)
$$$$
k=1,2, . ., r, \quad i=1,2, \ldots, m
$$

$\checkmark$ Se repiten los pasos dos, tres, y cuatro, para todos los patrones de entrenamiento.

$\checkmark$ Se repiten los pasos dos, tres, cuatro y cinco, hasta que la suma de los errores de todos los patrones se estabilice:

$$
e(n)=\frac{1}{2} \sum_{k=1}^{r}\left[y_{k}^{*}(n)-y_{k}(n)\right]^{2}
$$

\begin{tabular}{|c|c|c|c|}
\hline Fase & $\begin{array}{c}\text { Determinación de } \\
\text { los pesosy }\end{array}$ & Minimizar la & Mínimos \\
\hline Supervisada & umbrales de la red & función del error & cuadrados \\
\hline
\end{tabular}

Figura 6: Fase supervisada, de aprendizaje del método híbrido

\subsection{Evaluación del modelo de RBR}

\subsubsection{Validación cruzada}

Consiste en dividir los datos de la muestra en tres partes; una se utiliza como conjunto de entrenamiento para determinar los parámetros estimados del modelo de RBR; la segunda muestra denominada muestra de prueba es un conjunto independiente de datos que se utiliza para realizar un seguimiento de los errores durante el entrenamiento, con el fin de evitar un sobre entrenamiento de la red. La tercera muestra, denominada muestra de reserva, es otro conjunto independiente de datos que se utiliza para evaluar la RBR final; el error de la muestra de reserva proporciona una estimación "sincera" de la capacidad predictora del modelo de RBR.

\subsubsection{Clasificación}

Muestra una tabla de clasificación para cada variable dependiente categórica. Cada tabla proporciona el número de casos clasificados correcta e incorrectamente para cada categoria de variable dependiente. También se presenta el porcentaje de casos totales que se clasificaron correctamente. 
La salida deseada o regla de clasificación es:

$$
t_{i}(\mathbf{x})=\left\{\begin{array}{ll}
1, \text { si } & \mathbf{x} \in \mathbf{Y}_{i} \\
0, \text { si } & \mathbf{x} \notin \mathbf{Y}_{i}
\end{array} \quad i=1,2,3, \ldots \ldots ., q\right.
$$

\subsubsection{Curva COR}

Compara la tasa de falsos positivos con la de verdaderos positivos. Una curva COR proporciona una representación visual de la sensibilidad por 1-especificidad de todos los puntos de corte de clasificación posibles.

\subsection{Análisis de importancia de la variable explicativa}

Realiza un análisis de susceptibilidad, para calcular la importancia de cada variable explicativa en la determinación de la RBR. El análisis se basa en las muestras de entrenamiento y comprobación combinadas. Así se crea una tabla y un gráfico que muestra la importancia y la importancia normalizada de cada variable explicativa.

\subsection{Muestra, capa de entrada y sa-} lida

En el presente trabajo se utiliza el modelo de red de base radial para clasificar a los pobladores mayores de 14 años de tres distritos del Perú, de acuerdo a su percepción sobre los roles de género: más a los hombres, más a las mujeres, ambos por igual.

Trabajamos con la base de datos proporcionados por el MIMP (Ministerio de la Mujer y Poblaciones Vulnerables), data utilizada para la investigación VIOLENCIA FAMILIAR Y SEXUAL en mujeres y varones de 15 a 59 años, realizado en los distritos de San Juan de Lurigancho, Puno y Tarapoto, que contenía una muestra de 1775 entrevistados (890 hombres y 885 mujeres).

El tipo de muestreo utilizado fue probabilístico, trietápico e independiente por cada distrito.

El tamaño de la muestra total calculado con un nivel de confianza del 90,0 \%, un margen de error del 8,5\% y una tasa de no respuesta del 10,0\%.

La muestra se dividió en tres partes, ver Tabla 1; la distribución se realizó aplicando muestreo aleatorio simple.

Tabla1. Resumen del procesamiento de casos

\begin{tabular}{|l|c|c|}
\hline Muestra & n & Porcentaje \\
\hline Entrenamiento & 1241 & $69.9 \%$ \\
\hline Prueba & 280 & $15.8 \%$ \\
\hline Reserva & 254 & $14.3 \%$ \\
\hline Válidos & 1775 & $100.0 \%$ \\
\hline Excluidos & 0 & \\
\hline Total & 1775 & \\
\hline
\end{tabular}

La capa de entrada (variables explicativas):

$\mathbf{X}_{1}$ Trabaja actualmente.

$\mathbf{X}_{2}$ Nivel Educativo.

$\mathbf{x}_{3}$ Cuando era niña(o) recibió maltrato (físico y/o psicológico) por padres o personas que la(o) criaron.

$\mathbf{X}_{4}$ Existencia de situaciones violentas entre los padres de la entrevistada(o).

$\mathbf{x}_{5}$ Ha sufrido alguna forma de agresión sexual, antes de los 15 años.

$\mathbf{x}_{6}$ Ha sufrido agresión psicológica, por algún miembro de su familia en los últimos 12 meses. 
$\mathbf{X}_{7}$ Ha sufrido agresión fisica, por algún miembro de su familia en los últimos 12 meses.

$\mathbf{x}_{8}$ Ha sufrido agresión sexual, por algún miembro de su familia en los últimos 12 meses.

Los entrevistados recibieron los siguientes maltratos físicos y/o psicológicos, por sus padres o personas que los criaron: cachetadas, reprimendas verbales o gritos, patadas, puntapiés, cocachos, golpes con látigos o correas, jalones de cabello o de oreja, dejándolos encerrado, echándoles o hundiéndolos en agua; entre otros.

Las agresiones psicológicas frecuentes por algún miembro de su familia los últimos 12 meses: trato con insultos, burlas o desprecio, ignorar o rechazar, amenazas con hacerle daño fisico, prohibirle visitar a sus familiares y/o amistades, quitando o dañando sus pertenencias; entre otras.

Las agresiones fisicas frecuentes por algún miembro de su familia en los últimos 12 meses: cachetadas, tirones del cabello o lanzamientos contra la pared, patadas o puñetazos; entre otras.

Las agresiones sexuales frecuentes por algún miembro de su familia en los últimos 12 meses: manoseos, forzar a tener relaciones sexuales y amenazas o acciones de represalia por no aceptar tener relaciones sexuales; entre otras.

No se consideró al esposo(a)/conviviente, ex-esposo(a)/ ex-conviviente como miembro de la familia.

\section{La capa de salida (Percepción} acerca de los roles de género):

$\mathbf{Y}_{1}$ A quien le corresponde realizar las tareas del hogar.

$\mathbf{Y}_{2}$ A quien le corresponde mantener económicamente a la familia.

$\mathbf{Y}_{3}$ A quien le corresponde la educación de los hijos.

$\mathbf{Y}_{4}$ A quien le corresponde decidir sobre la sexualidad en la pareja.

Tareas del hogar: se consideraron cocinar, lavar la ropa, hacer compras.

Educación de los hijos: corregirlos, ocuparse de los asuntos escolares, atenderlos cuando se enferman y llevarlos para su atención, jugar y llevarlos a pasear, hablarles sobre sexualidad.

Sexualidad en la pareja: momento de tener relaciones, uso de anticonceptivos, decidir cuándo tener hijos y cuántos.

Las neuronas de la capa de entrada y salida se transformaron a una escala nominal binaria, en el caso de que las neuronas presentaban más de dos alternativas se utilizaron variables dummys.

\section{Resultados y discusión}

La Tabla 2 contiene la distribución de la muestra bajo estudio. La muestra total estaba conformada por:

$49,9 \%$ de mujeres y 50,1 \% de hombres; 33,1 \% de los entrevistados fueron del distrito de San Juan de Lurigancho, 33,1 \% de Puno y 33,8 \% de Tarapoto. 
Tabla 2. Distribución de la muestra

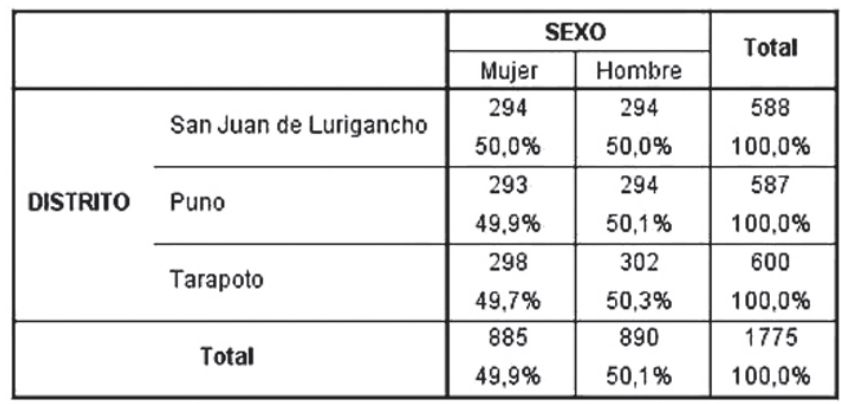

\subsection{Perfil de los entrevistados}

El 53,6 \% de las mujeres y el 72,1 $\%$ de los hombres se encontraban trabajando al momento de realizarse la entrevista.

Con respecto al nivel educativo alcanzado, el 48,2 \% de las mujeres alcanzó nivel secundario y 50,4 \% de los hombres educación superior.

$63,6 \%$ de las mujeres y 55,6 \% de los hombres se encuentra actualmente unidos.

$72,8 \%$ de las mujeres y 53,5 \% de los hombres tienen hijos.

$86,2 \%$ de las mujeres y el 92,1 $\%$ de los hombres sufrieron maltrato (físico y/o psicológico) cuando era niña(o) por sus padres o personas que la criaron.

47,6 \% de las mujeres y 52,1\% de los hombres manifestaron que hubo situaciones violentas entre sus padres.

$26,8 \%$ de las mujeres y el 20,6 \% de los hombres señaló que sufrió alguna forma de agresión sexual, antes de los 15 años.

9,8 \% de mujeres y 4,7 \% de hombres sufrieron agresión psicológica, por algún miembro de su familia en los últimos 12 meses.
3,3 \% de mujeres y 1,2\% de hombres manifestaron haber sufrido agresión física, por algún miembro de su familia en los últimos 12 meses.

$0,7 \%$ de mujeres y $2,8 \%$ de hombres no sufrieron agresión sexual, por algún miembro de su familia en los últimos 12 meses.

Con respecto a quien le corresponde realizar las tareas del hogar, el 75,4 $\%$ de las mujeres y el 65,7 \% de los hombres consideran a ambos géneros por igual.

El 79,7 \% de mujeres y 62,2 \% de los hombres consideran que mantener económicamente a la familia le corresponde tanto a hombres como a mujeres.

Respecto a quien debe ocuparse de la educación de los hijos, 94,6 \% de mujeres y 96,1 \% de hombres consideran que esta tarea la deben realizar mujeres y hombres.

Con respecto a las decisiones sobre la sexualidad de la pareja, el 94 \% y el 95,6 \% de mujeres y hombres respectivamente, consideran que las decisiones la deben tomar hombres y mujeres por igual. 


\subsection{Resultados del modelo}

La Tabla 3, muestra los resultados de la etapa de entrenamiento, prueba y reserva del modelo de red de base radial. Con los resultados del entrena- miento se estimarán los parámetros del modelo y con los de la etapa de reserva se evaluara la capacidad predictiva del modelo.

Tabla 3. Resumen del modelo

\begin{tabular}{|c|l|c|}
\hline \multirow{2}{*}{ Entrenamie nto } & Suma de errores cuadráticos & 619.021 \\
\cline { 2 - 3 } & $\begin{array}{l}\text { Porcentaje promedio de } \\
\text { pronósticos incorrectos }\end{array}$ & $17.0 \%$ \\
\hline \multirow{2}{*}{ Prueba } & Suma de errores cuadráticos & $137,542^{\mathrm{a}}$ \\
\cline { 2 - 3 } & $\begin{array}{l}\text { Porcentaje promedio de } \\
\text { pronósticos incorrectos }\end{array}$ & $17.2 \%$ \\
\hline \multirow{2}{*}{ Reserva } & $\begin{array}{l}\text { Porcentaje promedio de } \\
\text { pronósticos incorrectos }\end{array}$ & $17.4 \%$ \\
\hline
\end{tabular}

a. El número de unidades ocultas está determinado por el criterio de datos de prueba: El número "óptimo" de unidades ocultas es el que produce el menor error en los datos de prueba.

La figura 7, muestra la arquitectura de la red de base radial para nuestra investigación. Las ocho variables explicativas se refieren al estilo de crianza, relaciones familiares, situación laboral y nivel educativo; y las cuatro variables dependientes a la percepción sobre los roles de género que tienen los pobladores mayores de 14 años de los distritos de San Juan de Lurigancho, Puno y Tarapoto.

\subsection{Evaluación del modelo}

\subsubsection{Validación del modelo y cla- sificación}

Con el modelo obtenido en la etapa de entrenamiento, se procede a validarlo, para evaluar su capacidad de clasificación y predicción; utilizaremos el segundo (280) y tercer grupo (250) de datos. Los resultados se muestran en la Tabla 4.

Tabla 4. Porcentaje global de clasificación correcta

\begin{tabular}{|l|c|c|}
\hline \multicolumn{1}{|c|}{ Capa de salida } & Clasificación & Predicción \\
\cline { 2 - 3 } & Muestra prueba & Muestra de reserva \\
\hline $\mathrm{Y}_{1}:$ Tareas del hogar & $69,3 \%$ & $69,7 \%$ \\
\hline $\mathrm{Y}_{2}:$ Mantener económicamente a la familia & $71,1 \%$ & $71,7 \%$ \\
\hline $\mathrm{Y}_{3}:$ Educación de los hijos & $95,0 \%$ & $94,1 \%$ \\
\hline $\mathrm{Y}_{4}:$ Sexualidad de la pareja & $95,7 \%$ & $94,9 \%$ \\
\hline Porcentaje promedio & $\mathbf{8 2 , 8 \%}$ & $\mathbf{8 2 , 6 \%}$ \\
\hline
\end{tabular}


Los hombres y mujeres mayores de 14 años de los distritos de San Juan de Lurigancho, Puno y Tarapoto, fueron clasificados correctamente en un 69,3; 71,1; 95y 95,7 por ciento, con respecto a su percepción sobre a quién le corresponde realizar las tareas del hogar, mantener económicamente la familia, la educación de los hijos y decidir sobre la sexualidad en la pareja, respectivamente.

El porcentaje global de hombres y mujeres clasificados correctamente de acuerdo a su percepción acerca de los roles de género, es del 82,8\%; y del $82,6 \%$ para las predicciones.

Figura 7: Red de base radial con ocho variables explicativas y las cuatro variables dependientes.

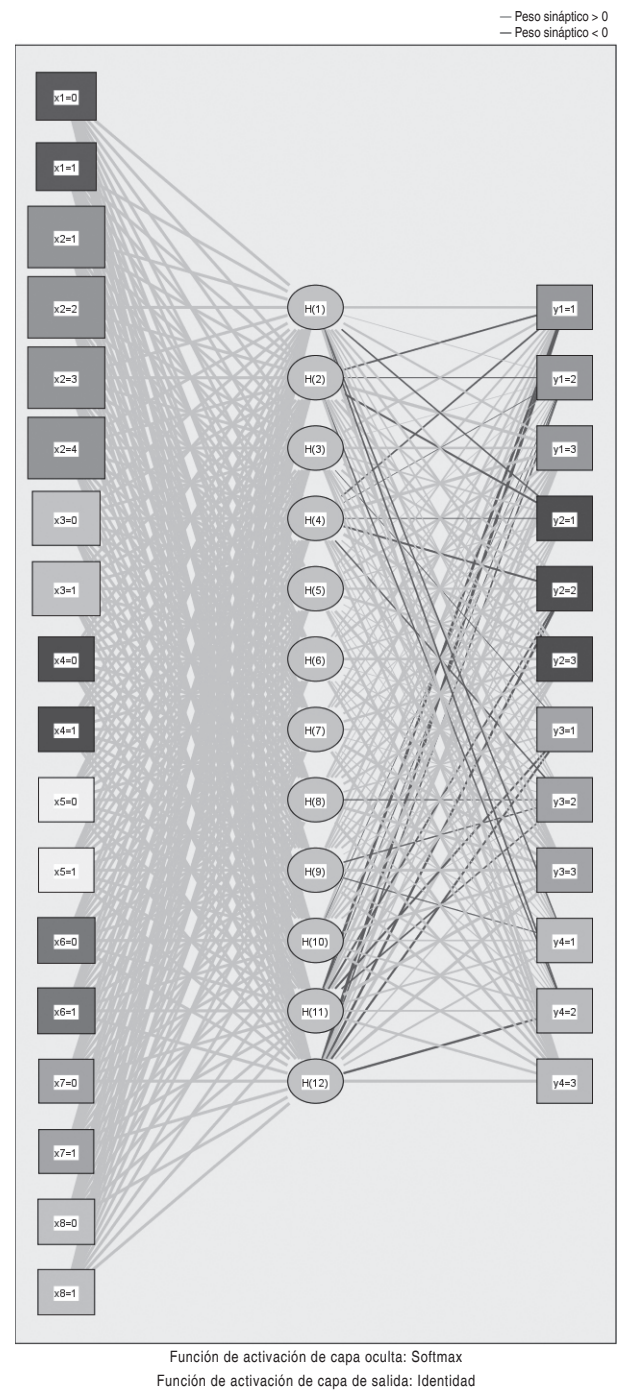




\subsubsection{Curva COR}

Las siguientes figuras muestran el comportamiento de la curva COR (características operativas del receptor) para cada una de las variables de respuesta del modelo red de base radial.
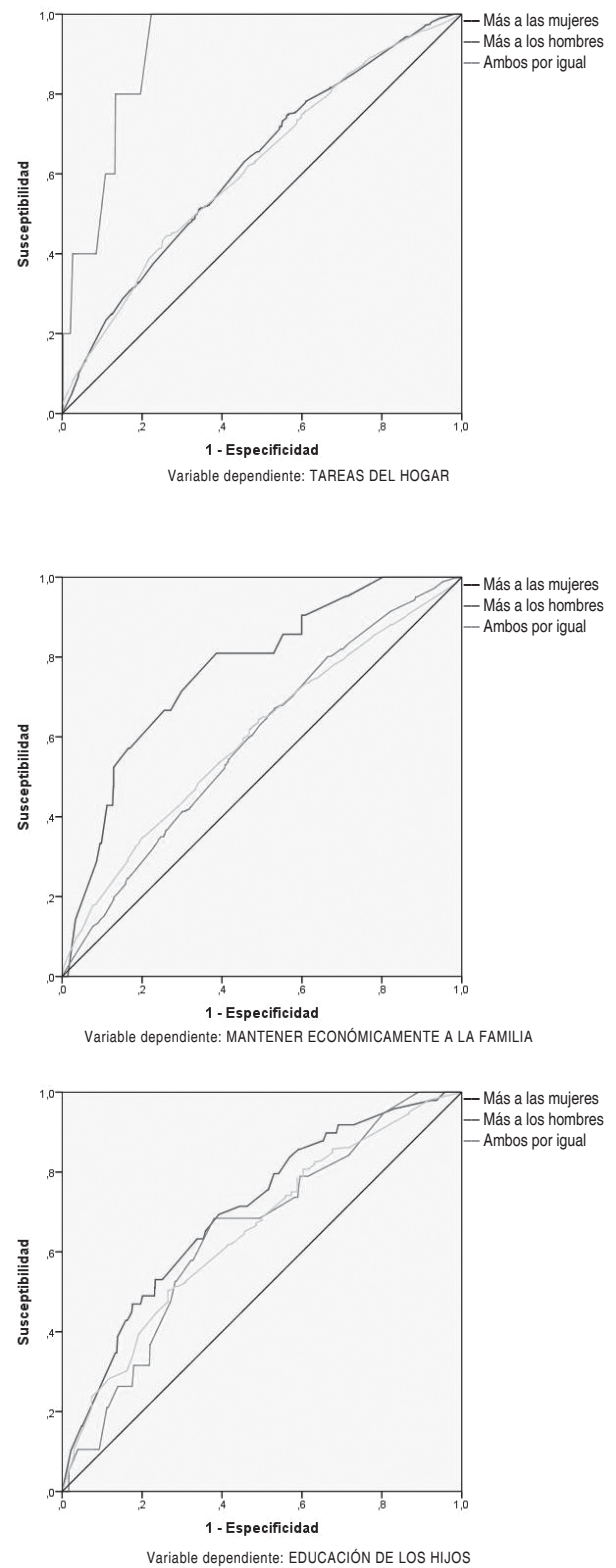

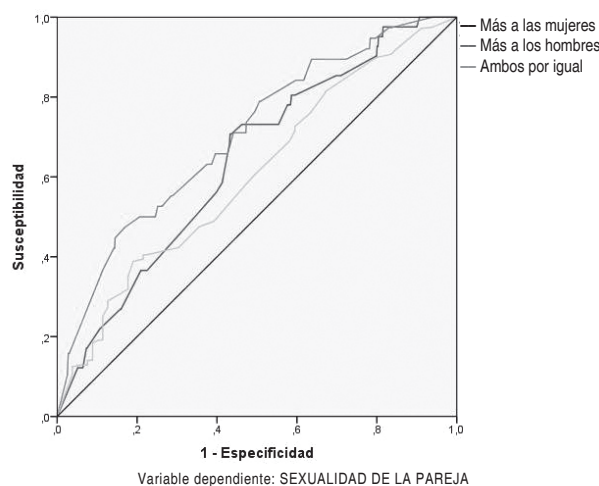

Podemos observar que las áreas bajo la curva son aceptables.

\subsection{Análisis de importancia de la variable explicativa}

La figura 9, presenta a las variables explicativas que más influyen en el modelo red de base radial:

$\mathrm{X}_{2}, \mathrm{X}_{3} \mathrm{y} \mathrm{X}_{1}$, es decir nivel educativo alcanzado, cuando era niña(o) recibió maltrato (físico y/o psicológico) por padres o personas que la(o) crió, y situación laboral.

Figura 9: Importancia de las variables explicativas en el modelo de RBR

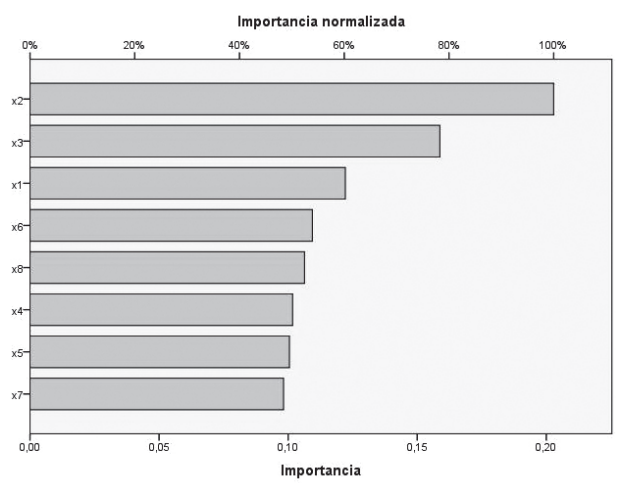




\section{Conclusiones}

1. El modelo red de base radial proporciona el $82,8 \%$ de clasificaciones correctas; es decir, aproximadamente 1470 de los 1775 entrevistados que residen en los distritos de San Juan de Lurigancho, Puno y Tarapoto, fueron clasificados de acuerdo a su percepción sobre el rol de género.

2. El nivel educativo alcanzado, cuando era niña(o) recibió maltrato (físico y/o psicológico) por padres o personas que la(o) criaron y la situación laboral, son los factores que más influyeron en la percepción sobre los roles de género, de hombres y mujeres mayores de 14 años, residentes en los distritos de San Juan de Lurigancho, Puno y Tarapoto.

3. Cuando se aplicó el modelo red de base radial por separado para hombres y mujeres, se obtuvo que los factores que más influyeron en la percepción sobre los roles de género de las mujeres son: nivel educativo, agresión psicológica en los últimos doce meses por algún miembro de su familia, y cuando era niña recibió maltrato (físico y/o psicológico) por padres o personas que la criaron; mientras que para el caso de los hombres: la situación laboral, haber sufrido alguna forma de agresión sexual antes de los 15 años y el nivel educativo alcanzado.

4. Las predicciones correctas sobre las percepciones sobre roles de género son del $82,6 \%$ de hombres y mujeres de los tres distritos. La mayoría considera que las tareas del hogar, mantener económicamente a la familia, la educación de los hijos y la sexualidad de la pareja, deben ser asumidos por igual entre hombres y mujeres.

5. Para lograr la igualdad y equidad de género, no es suficiente que se incremente porcentaje de niños que asisten a la escuela o que se den leyes para proteger a las mujeres; es necesario educar y reeducar a la familia; se requiere programas que logren transformar la cultura institucional.

\section{Agradecimiento}

A la Dra. Ana María Alejandra Mendieta Trefogli, Directora Ejecutiva del Programa Nacional contra la Violencia Familiar y Sexual del MIMP, por facilitarnos la base de datos con la que trabajamos en la presente investigación. 


\section{REFERENCIAS BIBLIOGRÁFICAS}

[1] Aldabas-Rubira, E. (2002) Introducción al reconocimiento de patrones mediante redes neuronales.

VII Jornadas de Conferencias - DEE- Campus Terrassa. Barcelona. Recuperado el 24 de enero del 2011, desde http://www.jcee.upc.edu/JCEE2002/ Aldabas.pdf

[2] Bardales, O., Huallpa, E. (2009) Violencia Familiar y Sexual en Mujeres y Hombres de 15 a 59 años: Estudio realizado en los distritos de San Juan de Lurigancho, Puno y Tarapoto. Lima: Ministerio de la Mujer y desarrollo Social.

[3] Bishop, C. (1995) Neural Networks for Pattern Recognition. United Kingdom. Oxford University Press.

[4] Haykin, S. (1999) Neural Networks a Comprehensive Foundation. 2. Ed. New Jersey: Prentice Hall International.

[5] Hernández, J., Ramírez, Mª J. y Ferri, C.(2005). Introducción a la Minería de Datos. Pearson Education S.A. Madrid.

[6] Hilera, J., Martinez, V. (1995) Redes Neuronales Artificiales. Fundamentos, modelos y aplicaciones. Madrid: Rama.

[7] Isasi, P., Galván, I. (2004) Redes de neuronas artificiales. Un enfoque práctico. Madrid: Pearson Prentice Hall.

[8] Pitarque, A., Roy, J., Ruiz, J. (1998) Redes neurales vs. Modelos estadísticos: Simulaciones sobre tareas de predicción y clasificación. Psicológica, 19, $387-400$. 